\author{
Jean-Michel Jeannin
}

Geschichte

Lange Zeit war die Verwendung der Kolanuss (botanisch: getrocknete Samen von Cola nitida und Cola acuminata) auf ihr Ursprungsgebiet Afrika beschränkt. Im 16. Jahrhundert wurde sie auch in Europa bekannt. 1865 wurde entdeckt, dass sie Koffein enthält [1]. Die Droge und ihre alkoholischen und wässrigen Extrakte waren im «National Formulary» der USA monographiert. Der Gehalt des Extrakts wurde nach Aromaintensität angegeben [2]. Nachdem sein erstes Getränk, Bordeauxwein mit einem Extrakt aus Kokablättern, wegen des Alkohols verboten wurde, entwickelte der amerikanische Apotheker Pemberton ein alkoholfreies, «medizinisch wertvolles Getränk». Die Kokablätter lieferten das Kokain, die Kolanüsse das Koffein. Die Zugabe von Kokain in Lebensmittel war um die vorletzte Jahrhundertwende nicht ungewöhnlich. Zum Kaschieren des bitteren Geschmacks fügte Pemberton dem Konzentrat Zucker hinzu und versetzte es mit Zitronen-

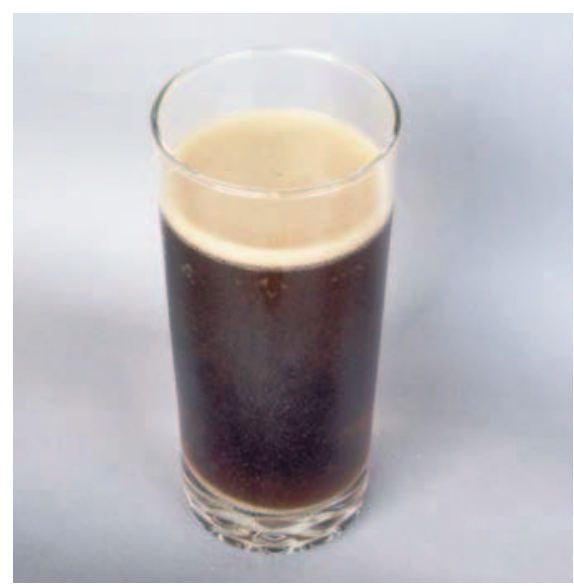

Abb. 1. Eine kühle Kolalimonade.

\section{Genussmittel und Ganzheitsmedizin (4)}

\author{
Kolanuss
}

säure. Weitere Zutaten sollen Orangen-, Muskat-, Zimt-, Limonen-, Koriander- und Neroliöl gewesen sein. Am 8. Mai 1886 wurde in der Jacob's Pharmacy in Atlanta zum ersten Mal Coca-Cola verkauft, das jeweils am Verkaufsort aus dem Konzentrat und Sodawasser zubereitet wurde. 1903 wurde das Kokain aus der Rezeptur entfernt, weil bekannt wurde, dass es süchtig macht. Man nimmt aber an, dass der Gehalt an Kokain ohnehin nur sehr niedrig war. Pemberton vermarktete sein Produkt als Heilmittel bei Kopfschmerzen und als Hilfe bei Nervenschwäche und Impotenz, aber auch als erfrischendes Stärkungsmittel «mit den Eigenschaften der wunderbaren Kokapflanze und der berühmten Kolanüsse». Sein Nachfolger Candler stellte 1899 auf Flaschenabfüllung um, wozu er die Rechte an zwei Unternehmer vergab, die ihm die Reinheit und Qualität des Produkts garantieren konnten. Das Geschäftsmodell wird heute noch praktiziert. Coca-Cola erhielt 1898 eine ebenfalls erfolgreiche Konkurrenz in Form der Pepsi-Cola, die ihren Namen vom Pepsin der Kolanuss und der Kolanuss selber erhielt. Sein Erfinder Caleb Bradham pries das Produkt als Mittel zur Förderung der Gesundheit an, z.B. im Bereich des Darms. Später wurden die Produkte nicht mehr als Heilmittel für eine kaufkräftige Gesellschaftsschicht, sondern als Erfrischungsgetränk für ein breites Publikum angepriesen (Abb. 1). Mittlerweile gibt es Kolagetränke ohne Zucker und ohne Koffein. Letzteres ist aber Bestandteil vieler sogenannter «Energy-Drinks» [3].

\section{C. nitida (Vent.) und C. acuminata} (P. Beauv.) Schott et Endl. (Sterculiaceae) Kola-Arten sind 10-20 m hohe immergrüne Bäume mit einer grauen Rinde und wechselständigen ledrigen Blättern, die sich am Ende abrupt verschmälern. Die weissen Blüten sind eingeschlechtlich, die länglichen, grünen und knotig-glatten Früchte hängen sternförmig angeordnet. Sie enthalten ledrige oder holzige Follikel, wovon jeder einen oder mehrere Samen enthält (Abb. 2). Wegen ihrer Konsistenz werden die getrockneten Samen auch Nüsse genannt $[1,2]$. Die Samen von C. nitida sind grösser als die von C. acuminata. Alle Kola-Arten sind in Westafrika heimisch [2].

\section{Chemie, Pharmakologie und Toxikologie der Kolasamen}

Kolasamen enthalten 1,5-2,0\% Koffein und geringe Mengen Theobromin. Weitere Bestandteile sind $d$-Catechin, $d l$-Catechin, $l$-Epicatechin, Betain, ein rotes Pigment, ein Glucosid, Tannine, 34\% Stärke, ca. 6,7\% Proteine sowie Fette, Zucker und Cellulose. Ein Teil des Koffeins ist an Catechin und/oder an Tannine gebunden [2]. Weiter enthalten die Samen Catechingerbstoffe, die beim Trocknen durch Polymerisation aus Catechin und Epicatechin entstehen. Wird weiter polymerisiert, entstehen Phlobaphene (auch: Kolarote) [4]. Die Samen wirken zentralnervös stimulierend, diuretisch, positiv inotrop, positiv chronotrop, glykogenolytisch, lipolytisch und atemanaleptisch [1]. Ferner stimulieren sie die Magensäuresekretion [5]. Diese Wirkungen werden durch das Koffein vermittelt. Koffein hemmt die cAMP-

\section{KARGER}

Fax +4976145207 14 Information@Karger.de www.karger.com
(๑) 2012 S. Karger GmbH, Freiburg
Dipl. med. biol. Jean-Michel Jeannin Holeestrasse 43, 4054 Basel, Schweiz

Tel. +41 61-4215991, Fax -4230313

jmjeannin@datanetworks.ch 


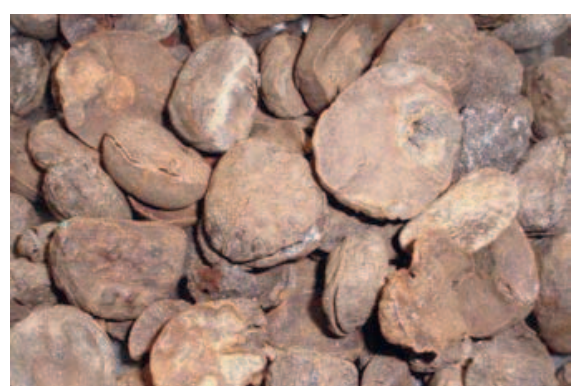

Abb. 2. Samen von verschiedenen KolaArten, mit und ohne Schale. Pharmaziehistorisches Museum Basel, 2012.

Phosphodiesterase und wirkt als Antagonist an den Adenosinrezeptoren. Das führt zu einer Freisetzung von Dopamin und zu einer Aktivierung zahlreicher Hirnareale. Die $\mathrm{LD}_{50}$ für Koffein beträgt $150-200 \mathrm{mg} / \mathrm{kg}$; für Kinder sind $5 \mathrm{~g}$ tödlich. Koffein kann zu einer Abhängigkeit führen [6]. Für das Wiederkehren des Wunsches nach Koffein ist das erlernte Wissen um seine stimulierende Wirkung, weniger die Substanz selber, verantwortlich («kognitive Abhängigkeit») [3].

\section{Erythroxylum coca}

E. coca ist ein 1,5 m hoher Strauch. Die unbehaarten Blätter sind dunkelgrün, die Blüten klein und weiss, die Steinfrüchte klein und rot. Es werden hauptsächlich je zwei Varietäten von E. coca und von $E$. novogranatense angebaut. Der Gesamtalkaloidgehalt der Blätter beträgt $0,1-1,4 \%$. Das wichtigste Alkaloid ist das Kokain. Es wirkt lokal betäubend und zentral euphorisierend. Die zentrale Wirkung wird durch eine erhöhte Freisetzung und eine Hemmung der Wiederaufnahme von Dopamin und Noradrenalin vermittelt [6].

\section{Verwendung von C. nitida und C. acuminata als Heilmittel}

In Afrika werden die Samen des Kolabaums traditionell gegen Hunger, Durst, Müdigkeit oder als anregendes Genussmittel gekaut. Beim Kauen verändert sich der bittere Geschmack durch Umwandlung von Stärke in Zucker und wird süss [7]. Die Kolasamen wurden medizinisch bei Migräne, bei Neuralgien, gegen Erbrechen und die Seekrankheit, bei Diarrhö sowie als Stimulans, Herztonikum, Aphrodisiakum und Diuretikum verwendet [1]. Gemäss dem Entwurf des Committee on Herbal Medicinal Products (HMPC) für eine Monographie über C. nitida und C. acuminata (P. Beauv.) Schott et Endl. semen wird die traditionelle Verwendung bei Erschöpfung und Schwächegefühl empfohlen [8]. Nach Schaffner et al. [1] sind die Samen von C. nitida und C. acuminata bei geistiger und körperlicher Ermüdung indiziert. Extractum Colae siccum oder fluidum sowie Tincturae oder Vinum colae werden als Anregungsmittel bei Müdigkeit und Abgespanntheit verabreicht [4]. Eine originelle Anwendung stellt die Schokolade mit Kolaextrakt dar [8]. In der Homöopathie wird Kola zur Behandlung des ADHS und des ADS sowie bei Schlafstörungen, Migräne, Heisshunger und Hungerattacken verwendet [8].

\section{Kulturelle Bedeutung}

Die Kolanüsse werden in ihrem Ursprungsgebiet als Zeichen der Freundschaft verschenkt [1]. Ausserhalb des Ursprungsgebiets hat sich keine dem Weingenuss vergleichbare Kultur ausgebildet. Der Konsum kolahaltiger Limonaden symbolisiert in hohem Mass die westliche und namentlich die amerikanische Lebensweise. Das ist nicht zuletzt der erfolgreichen aggressiven Werbung der beiden Marktführer zu verdanken [3]. Im Zentrum des Marketings steht die Mythenbildung um die geheime Rezeptur [3]. Mythen gehören klar auch zur modernsten Kultur. In der zweiten Hälfte des letzten Jahrhunderts waren die Kolalimonaden Teil der Jugendkultur und symbolisierten Abgrenzung und Auflehnung gegen die Erwachsenenwelt. Diese reagierte mit der Bildung von Legenden über die Gefährlichkeit der Kolagetränke, womit sich wieder einmal ein Kreis schloss ...

\section{Gesundheitlicher Nutzen der Kolanuss}

Der gesundheitliche Nutzen der Kolanuss kann in ihrer Funktion als mittlerweile traditionelles Heilmittel der Phytotherapie und Homöopathie gesehen werden.

\section{Risiken}

Die Risiken sind die eines übermässigen Gebrauchs von Koffein sowie einer übermässigen Kalorienzufuhr beim Konsum der zuckerhaltigen Limonaden mit Kolaextrakt. Der Gehalt an Koffein ist heutzutage gering; mit einer Suchtbildung muss nicht gerechnet werden.

\section{Schlussfolgerung}

Auch eine Limonade mit Kolageschmack, eisgekühlt an einem heissen Sommertag getrunken, kann ein Genuss sein. Man darf einfach das Koffein und den Zucker nicht vergessen.

\section{Dank}

Der Autor dankt dem Team des Pharmaziehistorischen Museums Basel für die Möglichkeit, Kolanüsse zu fotografieren, und weist gerne auf die Sonderausstellung «Kickstart - Koffein im Blut» (www.pharmaziemuseum.ch/) hin.

\section{Literatur}

1 Schaffner W: Heilpflanzen-Kompendium, ed 3. Hinterkappelen, Arboris, 1999.

2 Leung AY, Forster S: Encyclopedia of Common Natural Ingredients, ed 2. New York, Wiley, 1996.

3 Valerius-Mahler C: Kickstart. Koffein im Blut. Pharmaziehistorisches Museum Basel, 2012.

4 Frohe D: Heilpflanzenlexikon, ed 8. Stuttgart, WVG, 2006.

5 Weiss RF: Lehrbuch der Phytotherapie, ed 8. Stuttgart, Hippokrates, 1997.

6 Wink M, et al: Handbuch der giftigen und psychoaktiven Pflanzen. Stuttgart, WVG, 2008.

7 Stöcklin J: Kolanuss. Text zur Ausstellung «Wegzehrung». Universität Basel, 1996.

8 Committee on Herbal Medicinal Products (HMPC): Community herbal monograph on Cola nitida (Vent.) Schott et Endl. and its varieties and Cola acuminata (P. Beauv.) Schott et Endl. semen. 2011. 